\title{
Microsurgical Foraminotomy via Wiltse Paraspinal Approach for Foraminal or Extraforaminal Stenosis at L5-S1 Level : Risk Factor Analysis for Poor Outcome
}

\author{
Sung-lk Cho, M.D., ${ }^{1}$ Chung-Kee Chough, M.D., Ph.D., ${ }^{1}$ Shu-Chung Choi, M.D., Jin-Young Chun, M.D., Ph.D. ${ }^{2}$ \\ Departments of Neurosurgery, ${ }^{1}$ Anesthesiology and Pain Medicine, ${ }^{2}$ Yeouido St. Mary's Hospital, \\ The Catholic University of Korea College of Medicine, Seoul, Korea
}

Objective : The purpose of this study was to present the outcome of the microsurgical foraminotomy via Wiltse paraspinal approach for foraminal or extraforaminal (FEF) stenosis at L5-S1 level. We investigated risk factors associated with poor outcome of microsurgical foraminotomy at L5-S1 level. Methods : We analyzed 21 patients who underwent the microsurgical foraminotomy for FEF stenosis at L5-S1 level. To investigate risk factors associated with poor outcome, patients were classified into two groups (success and failure in foraminotomy). Clinical outcomes were assessed by the visual analogue scale (VAS) scores of back and leg pain and Oswestry disability index (ODI). Radiographic parameters including existence of spondylolisthesis, existence and degree of coronal wedging, disc height, foramen height, segmental lordotic angle (SLA) on neutral and dynamic view, segmental range of motion, and global lumbar lordotic angle were investigated.

Results : Postoperative VAS score and ODI improved after foraminotomy. However, there were 7 patients (33\%) who had persistent or recurrent leg pain. SLA on neutral and extension radiographic films were significantly associated with the failure in foraminotomy $(p<0.05)$. Receiver-operating characteristics curve analysis revealed the optimal cut-off values of SLA on neutral and extension radiographic films for predicting failure in foraminotomy were $17.3^{\circ}$ and $24^{\circ} \mathrm{S}$, respectively.

Conclusion : Microsurgical foraminotomy for FEF stenosis at L5-S1 level can provide good clinical outcomes in selected patients. Poor outcomes were associated with large SLA on preoperative neutral $\left(>17.3^{\circ}\right)$ and extension radiographic films $\left(>24^{\circ}\right)$.

Key Words : Lumbar vertebrae · Foraminal stenosis · Foraminotomy $\cdot$ L5 root $\cdot$ Lordosis.

\section{INTRODUCTION}

Lumbar foraminal or extraforaminal (FEF) stenosis is a common cause of lumbar radiculopathy, with a reported incidence rate of $8-11 \%^{11,14,19)}$. There are two surgical treatment options for lumbar FEF stenosis : decompression without fusion and decompression with spinal fusion ${ }^{1-3,8,11-14,16,17,20)}$.

In 1988, Wiltse and Spencer introduced microsurgical decompression of FEF lesions through so called "the Wiltse paraspinal approach" to the lumbar spine ${ }^{21)}$. After this description, many surgeons have modified this paraspinal approach to obtain the direct access to far lateral lesions, minimizing violation of the facet joint ${ }^{3)}$. The reported success rate following microsurgical foraminotomy of FEF lesions is ranging from 58 to $80 \%^{1,2,4-7,9,12,14)}$, but many patients have postoperative leg pain, which is the main cause of poor outcomes ${ }^{2,4-6,9,14)}$. Some prominent previous studies reported relatively high rate of persistent or recurrent radiculopathy after microsurgical foraminotomy, especially after surgery at the L5-S1 level ${ }^{2,3,22}$. The persistent or recurrent radiculopathy rate was higher in patients with FEF stenosis at L5-S1 level than patients with FEF stenosis at other than L5-S1 level, but there have been only a few studies on preoperative risk factors for persistent or recurrent radiculopathy ${ }^{2,3,22)}$.

Therefore, a clinical analysis was performed to present the outcome of the microsurgical foraminotomy for FEF stenosis at L5-S1 level. Also, we retrospectively investigated preoperative risk factors (demographic, clinical, and radiographic) associated with persistent or recurrent radiculopathy after microsurgical foraminotomy at L5-S1 level.

- Received : September 29, 2016 • Revised : October 6, 2016 • Accepted : October 7, 2016

- Address for reprints : Chung-Kee Chough, M.D., Ph.D.

Department of Neurosurgery, Yeouido St. Mary's Hospital, The Catholic University of Korea College of Medicine, 10 63-ro, Yeongdeungpo-gu, Seoul 07345, Korea

Tel : +82-2-3779-1187, Fax : +82-2-786-5809, E-mail : chough65@gmail.com

- This is an Open Access article distributed under the terms of the Creative Commons Attribution Non-Commercial License (http://creativecommons.org/licenses/by-nc/3.0) which permits unrestricted non-commercial use, distribution, and reproduction in any medium, provided the original work is properly cited. 


\section{MATERIALS AND METHODS}

\section{Patient's data and study design}

This study included 21 consecutive patients having undergone microsurgical foraminotomy for FEF stenosis at L5-S1 between 2010 and 2014 at our institution. The operative indication was combination of chronic mono-radiculopathy resistant to conservative treatment, lateral sagittal and coronal T2-weighted MRI images showing FEF stenosis of unilateral L5 nerve, and plain X-ray films showing at the L5-S1 level : $<20^{\circ}$ Cobb angle (tilting angle of L5 against sacrum), <2 mm lateral slip. The operative contraindications were patients with : 1) definite segmental instability on dynamic radiographs, 2) spondylolisthesis over grade 1 , and 3) degenerative lumbar scoliosis $>20^{\circ}$ Cobb angle.

The medical records of these patients were reviewed for demographic data, preoperative clinical symptom, and postoperative clinical course. The study excludes subjects with :1) a history of previous lumbar operation, 2) multilevel surgeries, and 3) less than 12 months of follow-up period. Because of the very selective indication, the patients were recruited over a 4-year timespan.

For clinical assessment, we obtained visual analog scale (VAS) scores of back pain (VAS-b), VAS scores of leg pain (VAS-l) and Oswestry disability index (ODI) at preoperative and postoperative period.

Because leg pain is a typical and representing symptom of radiculopathy ${ }^{3)}$, we divided patients into two groups according to postoperative leg pain. The foraminotomy failure (FF) group included patients that postoperative VAS-l was over 3 , and the other patients that postoperative VAS-l was same or less than 3 were classed as the foraminotomy success (FS) group.

For evaluation of radiographic parameters, preoperative simple lumbosacral radiographs with dynamic views were accessed. Segmental lordotic angle (SLA) at the L5-S1 level, global lumbar lordotic angle, and coronal wedging angle were calculated. The degree of coronal wedging at L5-S1 was noted as angle between the lines drawn through the superior endplates of $\mathrm{L} 5$ and $\mathrm{S}^{15}{ }^{15}$. Preoperative CT scans with coronal and sagittal reconstruction were reviewed for existence of spondylolisthesis and accurate measurement of disc and foraminal heights. All measurements were made using a picture-archiving and communications system (Maroview, version 5.4; Marotech, Inc., Seoul, Korea).

\section{Surgical procedures}

All operations were performed by one senior author. Briefly, All patients were operated while prone and under general anesthesia. Under fluoroscopic guidance, a skin incision of $3.5 \mathrm{~cm}$ was made 3-4 cm lateral to the spinous processes, and the FEF zone (L5 transverse process and isthmus, sacral ala, and lateral margin of the L5-S1 facet) was exposed via the intermuscular approach proposed by Wiltse et al. $^{21)}$. After muscle dissection, a self-retaining retractor and surgical microscope were set up. The superolateral part of the superior articular process of S1 and the lower border of the L5 transverse process were resected using a high-speed drill. Tilting the surgical microscope to the medial side, the lateral part of the isthmus and the superomedial part of the superior articular process were resected. After confirming the upper pedicle, the intertransverse and foraminal ligament was dissected and excised. Then, the affected L5 nerve root was exposed. With thin-foot-plate punches, decompression was continued medially towards the lateral rim of the yellow ligament. Sufficient nerve root decompression was carefully confirmed by dissectors. If necessary, the disc space was exposed, and discectomy was performed for complete decompression. If patients had extraforaminal stenosis, additional extraforaminal decompression was performed by tilting the surgical microscope to the lateral side : the lower part of the L5 transverse process, superomedial part of the sacral ala, and lumbosacral ligament were resected. After decompression, the wound was closed in layered fashion.

\section{Factors investigated in association with poor outcome}

The demographic and clinical parameters included age, gender, side of L5 radiculopathy, VAS-b, VAS-l, and ODI. The preoperative radiologic parameters included existence of spondylolisthesis, existence and degree of coronal wedging, disc height, foramen height, SLAs in neutral and dynamic view, segmental range of motion, and global lumbar lordotic angle. Spondylolisthesis was present when more than $4 \mathrm{~mm}$ slip in the lateral radiographic film.

\section{Statistical analysis}

Statistical analysis was performed using SPSS statistical software package 18.0 for Windows (SPSS Inc, Chicago, IL, USA). The independent t-test and Fisher's exact test were appropriately used to analyze the differences and evaluate the statistical significance, which was set at the $p=0.05$ level. For evaluating predictive factors associated with poor outcome, receiver-operating characteristics (ROC) curve was analyzed and 95\% confidence interval (CI) was calculated. Cut-off value was determined maximized value in sum of sensitivity and specificity.

\section{RESULTS}

There were 9 men and 12 women. The mean follow up periods were 18 months (12-24 months). The mean age at the time of surgery was 67.2 (range, 47-82 years). All patients presented with leg pain in the distribution of the L5 nerve root localized to one side (12 right, 9 left). Mean preoperative VAS-1 was 6.1 (range, 3-9). Also, the majority of patients complained of low back pain. Mean preoperative VAS-b and ODI were 4.2 (range, 2-8) and 62 (range, 26-100), respectively. Preoperative motor weakness was noted in one patient. Postoperative leg pain was improved in all patients. Mean postoperative VAS-1 was 2.6 (range, 0-7). Postoperative VAS-b and ODI were not aggravated in all patients. Mean postoperative VAS-b and ODI were 2.6 (range, 
0-6) and 26.8 (range, 8-66), respectively.

Seven patients were classed as the FF group that postoperative VAS-l was over 3. There were 14 patients in the FS group. Demographics and perioperative clinical parameters of each group were summarized in Table 1 . There were no significant difference between the two groups in terms of demographic and perioperative clinical parameters except for postoperative ODI. Postoperative ODI was significantly higher in the FF group. We also investigated the influence of discectomy at the time of surgery

Table 1. Demographics and perioperative clinical parameters of patients according to subgroup

\begin{tabular}{lccc}
\hline \multicolumn{1}{c}{ Parameters } & FF group & FS group & $p$-value \\
\hline Number of patients & 7 & 14 & \\
Sex (male/female) & $4 / 3$ & $5 / 9$ & 0.397 \\
Age & $66.6 \pm 7.4$ & $67.6 \pm 10.5$ & 0.825 \\
Side (right/left) & $3 / 4$ & $9 / 5$ & 0.397 \\
Discectomy & 2 & 8 & 0.361 \\
Preop VAS-1 & $6.9 \pm 1.3$ & $5.7 \pm 1.2$ & 0.063 \\
Preop VAS-b & $4 \pm 1.5$ & $4.3 \pm 1.6$ & 0.697 \\
Preop ODI & $58.9 \pm 21.8$ & $63.6 \pm 20.8$ & 0.643 \\
Postop VAS-b & $3.5 \pm 1.1$ & $2.1 \pm 1.7$ & 0.053 \\
Postop ODI & $40.3 \pm 18.9$ & $20 \pm 13.3$ & 0.009 \\
\hline
\end{tabular}

Values are mean \pm SD. VAS-I : visual analogue scale score of leg pain, VAS-b : visual analogue scale score of back pain, ODI : Oswestry disability index, FF : foraminotomy failure, FS : foraminotomy success

Table 2. Preoperative radiographic parameters associated foraminotomy failure

\begin{tabular}{lccc}
\hline \multicolumn{1}{c}{ Parameters } & FF group & FS group & p-value \\
\hline Spondylolisthesis & 1 & 3 & 1.0 \\
Coronal wedging & 2 & 4 & 1.0 \\
Coronal wedging angle $\left(^{\circ}\right)$ & $2 \pm 3.6$ & $3.5 \pm 7$ & 0.605 \\
Posterior disc height $(\mathrm{mm})$ & $6.7 \pm 2.2$ & $6.7 \pm 2.3$ & 0.99 \\
Foramen height $(\mathrm{mm})$ & $14.9 \pm 1.2$ & $16.3 \pm 3.1$ & 0.146 \\
Neutral SLA $\left(^{\circ}\right)$ & $18.4 \pm 4.4$ & $13 \pm 4.7$ & 0.02 \\
Flexion SLA $\left(^{\circ}\right)$ & $14.6 \pm 5.4$ & $10.1 \pm 7.1$ & 0.24 \\
Extension SLA $\left({ }^{\circ}\right)$ & $24.8 \pm 4.2$ & $17.9 \pm 5$ & 0.005 \\
Segmental range of motion $\left(^{\circ}\right)$ & $10.2 \pm 3.9$ & $10.8 \pm 4.8$ & 0.787 \\
Global lumbar lordotic angle $\left(^{\circ}\right)$ & $4.7 \pm 56.1$ & $23 \pm 10.9$ & 0.422 \\
\hline V
\end{tabular}

Values are mean \pm SD. SLA : segmental lordotic angle, FF : foraminotomy failure, FS : foraminotomy success on outcome, but discectomy did not affect outcome of surgery. Statistical analysis of preoperative radiographic parameters revealed that SLAs on neutral and extension radiograph films were significantly larger in the FF group than the FS group. In the FF group, the mean SLA on neutral and extension radiographic films were $18.4^{\circ}$ and $24.8^{\circ}$, respectively. In the FS group, the mean SLA on neutral and extension radiographic films were $13^{\circ}$ and $17.9^{\circ}$, respectively. Other radiographic parameters did not show any significant differences (Table 2). The ROC curve analysis was used to determine the optimal cut-off values of the SLA on neutral and extension radiographic films for predicting failure in foraminotomy. When SLA on neutral radiographic film was less than $17.3^{\circ}$, the area of FF group was largest. The area was 0.786 ( $p=0.015,95 \%$ CI $0.554-0.932$ ), sensitivity was $100 \%$ and specificity was $57.14 \%$. When SLA on extension radiographic film was less than $24^{\circ}$, the area of FF group was largest. The area was 0.878 ( $p<0.001,95 \%$ CI $0.662-0.978)$, sensitivity was $100 \%$ and specificity was $71.43 \%$. In other words, SLA larger than $17.4^{\circ}$ on neutral and $24^{\circ}$ on extension radiographic film were associated with poor outcome after microsurgical foraminotomy at L5-S1 level.

In the FF group, revision surgeries were performed in 2 of 7 patients ( 2 months after the initial surgery). Posterior lumbar interbody fusion was selected for direct root decompression and stabilization. Nerve root blocks were performed in two patients, and others were treated with oral medication for pain relief (Table 3).

\section{DISCUSSION}

Since its introduction in $1988^{21)}$, microsurgical foraminotomy via Wiltse paraspinal approach has been regarded as a principal surgical option for lumbar FEF lesions without instability and deformity. Reported success rate of microsurgical foraminotomy for FEF lesions is ranging from 58 to $80 \%^{1,2,4-7,9,12,14,18,22)}$. However, some previous studies demonstrated higher poor outcome rates of microsurgical foraminotomy for FEF lesions of lumbar spine than other forms of spine surgery and other regions of lumbar spine $e^{2,3,22)}$.

Chang et al. ${ }^{3)}$ reported that $21.7 \%$ of patients had persistent or recurrent leg pain after lumbar FEF microdecompression. Bae

Table 3. Summary of cases of foraminotomy failure group

\begin{tabular}{ccccccccccc}
\hline No. & Age & Sex & Side & Prepop VAS-1 & Postop VAS-1 & Postop ODI & Postop VAS-b & Neutral SLA & Extension SLA & Treatment \\
\hline 1 & 55 & M & R & 6 & 5 & 66 & 4 & 13.4 & 16.9 & PLIF \\
2 & 73 & F & R & 7 & 6 & 10 & 3 & 24.5 & 26.8 & Nerve block \\
3 & 69 & F & L & 8 & 4 & 38 & 3 & 13.2 & 26.1 & Nerve block \\
4 & 58 & M & R & 5 & 4 & 52 & 5 & 21.3 & 22.6 & Medication \\
5 & 71 & M & L & 6 & 4 & 36 & 2 & 21.3 & 25 & Medication \\
6 & 66 & F & L & 9 & 4 & 52 & 5 & 19.7 & 26.4 & Medication \\
7 & 74 & M & L & 7 & 6 & 28 & 3 & 15.3 & 30.1 & PLIF \\
\hline
\end{tabular}

No : number, Preop : Preoperative, VAS-I : visual analogue scale score of leg pain, Postop : Postoperative, ODI : Oswestry disability index, VAS-b : visual analogue scale score of back pain, SLA : segmental lordotic angle, R : right, L : left, PLF : posterior lumbar interbody fusion 
et al. ${ }^{2)}$ and Yamada et al. ${ }^{22)}$ reported poor outcome rate of $22.2 \%$ and $19.6 \%$, respectively. In these 3 studies, poor outcomes and revision surgeries were more common at L5-S1 level than other level of lumbar spine. Bae et al. ${ }^{2)}$ presented that $47.1 \%$ of patients at L5-S1 level had pair and poor outcome which was higher than other level and 10 of 13 (77\%) revision surgeries were performed at L5-S1 level. In the other study, 5 of 9 (55\%) revision surgeries were performed at L5-S1 level ${ }^{3)}$. Our study showed poor outcome in $33 \%$ patients. The incidence of revision surgery in this study was $10 \%$, which was comparable to that of previous studies $2,3,13,16-18,22)$.

In our study, there was a significant difference of postoperative ODI between FF and FS groups $(p=0.009)$. However, postoperative VAS-b did not show a significant difference between two groups. We assumed that this result came from influence of the postoperative leg pain on the postoperative ODI.

There have been only a few reports on the risk factors following microsurgical foraminotomy for FEF lesions ${ }^{2,3,22)}$. Chang et al. ${ }^{3)}$ presented that the patients with double disc herniation (combination of intracanalicular and far lateral disc at the same level) were almost three times more likely to have remaining or recurrent leg pain than the patients who required paraspinal approach only. Bae et al. ${ }^{2)}$ reported that the amount of facet removal was closely related to clinical outcomes of the operation for FEF disc herniation. The reason for poor outcome in both studies was that double disc herniation requiring combined discectomy (intracanalicular and paraspinal approach simultaneously) made great facet resection and annular removal which could cause segmental instability and disc space collapse ${ }^{2,3)}$. However, extraforaminal disc herniations at L5-S1 are very rare and the incidence of compression of the $\mathrm{L}-5$ nerve at the extraforaminal region of L5-S1 is probably higher than that accounted for by disc herniations alone ${ }^{15}$. Our study did not include any case of double disc herniation. Therefore, as a risk factor, double disc herniation was not within the scope of our study.

Yamada et al. ${ }^{22)}$ suggested degenerative lumbar scoliosis as a risk factor for poor outcome following microsurgical foraminotomy of lumbar spine. They reported that the recurrence rate was significantly higher and the clinical improvement was significantly smaller in patients with degenerative lumbar scoliosis than in those without degenerative lumbar scoliosis. Our series did not include patients with degenerative lumbar scoliosis $>20^{\circ}$ to minimize influence of scoliosis. Coronal wedging is probably a more significant finding at L5-S1 than at upper lumbar levels due to the narrow extraforaminal space. We expected that coronal wedging would be a risk factor for poor outcome. However, existence and degree of coronal wedging did not show any significant differences. Previous studies also failed to demonstrate statistical significance of coronal wedging ${ }^{2,22)}$.

In our study, large SLA on neutral and extension films were associated with poor outcome of microsurgical foraminotomy at L5-S1 level. Previous studies reported that segmental angle and flexion-extension movement affect intervertebral foramen size and volume ${ }^{1,2)}$. Inufusa et al. ${ }^{10)}$ demonstrated that the crosssectional area of foramen to be $15 \%$ smaller for the extension group than the cross-sectional area of the neutral group. Facet removal and additional bone resection at the already lordotic L5-S1 segment may cause sagittal instability and further increase of SLA. Combination of the sagittal instability and increased SLA may result in the progression of FEF stenosis. In case of FEF stenosis at L5-S1 with large SLA, direct and indirect decompression of L5 root combined with various fusion techniques would provide better surgical outcome than foraminotomy alone.

Generally speaking, most spine surgeons tend to pay more attention to scoliosis and coronal instability than lordosis and sagittal instability when they are dealing with lumbar FEF stenosis. From our results, the SLA should be taken into consideration when we are dealing with FEF stenosis at L5-S1 level.

The biggest weakness of our study is a small number of the patients included. However, it was very difficult to recruit the patients who met the inclusion criteria of this study. Further experience and multi-center collaboration will elucidate the definitive risk factors for poor outcome following microsurgical foraminotomy for FEF stenosis at L5-S1 level.

\section{CONCLUSION}

FEF stenosis at L5-S1 is not common but important cause of lumbar radiculopathy. FEF stenosis at L5-S1 level is different from FEF stenosis at upper lumbar level in terms of anatomy and pathology. Microsurgical foraminotomy via Wiltse approach for FEF stenosis at L5/S1 can provide good clinical outcomes in selected patients. But, poor outcomes are associated with large lordotic angles at preoperative neutral (over $17.3^{\circ}$ ) and extension radiograph (over $24^{\circ}$ ).

\section{References}

1. Baba H, Uchida K, Maezawa Y, Furusawa N, Okumura Y, Imura S : Microsurgical nerve root canal widening without fusion for lumbosacral intervertebral foraminal stenosis : technical notes and early results. Spinal Cord 34 : 644-650, 1996

2. Bae JS, Kang KH, Park JH, Lim JH, Jang IT : Postoperative clinical outcome and risk factors for poor outcome of foraminal and extraforaminal lumbar disc herniation. J Korean Neurosurg Soc 59 : 143-148, 2016

3. Chang SB, Lee SH, Ahn Y, Kim JM : Risk factor for unsatisfactory outcome after lumbar foraminal and far lateral microdecompression. Spine (Phila Pa 1976) 31 : 1163-1167, 2006

4. Darden BV 2nd, Wade JF, Alexander R, Wood KE, Rhyne AL 3rd, Hicks JR : Far lateral disc herniations treated by microscopic fragment excision. Techniques and results. Spine (Phila Pa 1976) 20 : 1500-1505, 1995

5. Donaldson WF 3rd, Star MJ, Thorne RP : Surgical treatment for the far lateral herniated lumbar disc. Spine (Phila Pa 1976) 18 : 1263-1267, 1993

6. Epstein NE : Foraminal and far lateral lumbar disc herniations : surgical alternatives and outcome measures. Spinal Cord $40:$ 491-500, 2002

7. Gioia G, Mandelli D, Capaccioni B, Randelli F, Tessari L : Surgical treatment of far lateral lumbar disc herniation. Identification of compressed root and discectomy by lateral approach. Spine (Phila Pa 1976) 24 : 1952 1957, 1999 
8. Hallett A, Huntley JS, Gibson JN : Foraminal stenosis and single-level degenerative disc disease : a randomized controlled trial comparing decompression with decompression and instrumented fusion. Spine (Phila Pa 1976) 32 : 1375-1380, 2007

9. Hodges SD, Humphreys SC, Eck JC, Covington LA : The surgical treatment of far lateral L3-L4 and L4-L5 disc herniations. A modified technique and outcomes analysis of 25 patients. Spine (Phila Pa 1976) 24 : 1243-1246, 1999

10. Inufusa A, An HS, Lim TH, Hasegawa T, Haughton VM, Nowicki BH : Anatomic changes of the spinal canal and intervertebral foramen associated with flexion-extension movement. Spine (Phila Pa 1976) 21 : 24122420, 1996

11. Jenis LG, An HS : Spine update. Lumbar foraminal stenosis. Spine (Phila Pa 1976) 25 : 389-394, 2000

12. Jenis LG, An HS, Gordin R : Foraminal stenosis of the lumbar spine : a review of 65 surgical cases. Am J Orthop (Belle Mead NJ) 30 : 205-211, 2001

13. Kim HJ, Jeong JH, Cho HG, Chang BS, Lee CK, Yeom JS : Comparative observational study of surgical outcomes of lumbar foraminal stenosis using minimally invasive microsurgical extraforaminal decompression alone versus posterior lumbar interbody fusion : a prospective cohort study. Eur Spine J 24 : 388-395, 2015

14. Kunogi J, Hasue M : Diagnosis and operative treatment of intraforaminal and extraforaminal nerve root compression. Spine (Phila Pa 1976) 16 : 1312-1320, 1991
15. Lee S, Kang JH, Srikantha U, Jang IT, Oh SH : Extraforaminal compression of the L-5 nerve root at the lumbosacral junction : clinical analysis, decompression technique, and outcome. J Neurosurg Spine 20 : 371-379, 2014

16. Matsumoto M, Watanabe K, Ishii K, Tsuji T, Takaishi H, Nakamura M, et al. : Posterior decompression surgery for extraforaminal entrapment of the fifth lumbar spinal nerve at the lumbosacral junction. J Neurosurg Spine 12 : 72-81, 2010

17. Ozeki N, Aota Y, Uesugi M, Kaneko K, Mihara H, Niimura T, et al. : Clinical results of intrapedicular partial pediculectomy for lumbar foraminal stenosis. J Spinal Disord Tech 21 : 324-327, 2008

18. Papavero L, Kothe R : Microsurgical extraforaminal decompression of lumbar root canal stenosis. Oper Orthop Traumatol 25 : 16-30, 2013

19. Porter RW, Hibbert C, Evans C: The natural history of root entrapment syndrome. Spine (Phila Pa 1976) 9: 418-421, 1984

20. Spivak JM, Kummer FJ, Chen D, Quirno M, Kamerlink JR : Intervertebral foramen size and volume changes in low grade, low dysplasia isthmic spondylolisthesis. Spine (Phila Pa 1976) 35 : 1829-1835, 2010

21. Wiltse LL, Spencer CW : New uses and refinements of the paraspinal approach to the lumbar spine. Spine (Phila Pa 1976) 13 : 696-706, 1988

22. Yamada K, Matsuda H, Nabeta M, Habunaga H, Suzuki A, Nakamura $\mathrm{H}$ : Clinical outcomes of microscopic decompression for degenerative lumbar foraminal stenosis : a comparison between patients with and without degenerative lumbar scoliosis. Eur Spine J 20 : 947-953, 2011 